\title{
Perspectivas de la ficología en México
}

\author{
MARTHA M. ORTEGA Y JOSE LUIS GODINEZ \\ Depto. de Botánica, Instituto de Biología, UNAM. Apdo. Postal 70-233, Coyoacán. 04510 México, D.F.
}

\begin{abstract}
Resumen. En este trabajo se presenta un visión panorámica sobre el quehacer ficológico en México durante la última década de este siglo, y sus perspectivas para los próximos años. El estudio se realizó revisando los foros científicos del país relacionados con la ficología. No obstante que la labor ficológica desempeñada es muy vasta, los estudios florísticos o la exploración algológica continuan siendo el tema central de la ficología nacional. Se observa que la ficología va en aumento por el crecimiento de su infraestructura humana e institucional.
\end{abstract}

Abstract. In this paper a general view on the phycologic studies in Mexico of the last decade of this century and their perspectives for the near future is presented. The paper was done reviewing the national forums related with the phycology. The phycologic work is meritorious, but the floristic research is getting along as the main subject. The mexican phycology is developing with the support of human and institutional support.

\section{INTRODUCCIÓN}

En las actuales circunstancias de desarrollo de la ficología en México, resulta útil reflexionar sobre las perspectivas de esta área de estudio. Se presenta una visión panorámica de la ficología nacional a manera de un conteo rápido, analizando su desarrollo y reflexionando sobre lo que esperamos de esta área en las próximas décadas. Este trabajo recoge principalmente las experiencias de los autores, además de lo consultado en algunas publicaciones relacionadas con el tema, con el fin de contar con datos aproximados a la realidad del país.

En primer lugar se reflexiona sobre la ficología en el mundo, a fin de tener un marco de referencia sobre las disciplinas que con mayor frecuencia se han abordado en otros países. Posteriormente se analiza la ficología nacional y las disciplinas que ha emprendido. Una forma rápida de observar este fenómeno es a través de los foros científicos recientes realizados en México -de la última década de este siglo- con el propósito de entresacar algunas realidades de la ficología mexicana, resaltando la infraestructura en sus aspectos humanos e institucionales y finalmente realizando un análisis de esas realidades y lo que esperamos de ellas en los próximos años.

\section{LA FICOLOGÍA MUNDIAL Y SUS ÁREAS DE ESTUDIO}

El alcance de la ficología es verdaderamente enorme, como se desprende de la revisión de títulos y resúmenes de un período que abarca de 1990 a 1994 de algunas revistas ficológicas de corte internacional, como Phycologia, Journal of Phycology, Botanica Marina y Criptogamie. En la Fig. 1 se aprecian las áreas de estudio que se desarrollaron principalmente en los últimos años. Siguen siendo del quehacer ficológico la taxonomía, la morfología, los estudios florísticos -incluyendo catálogos- y la ecología, además de algunas áreas de sofisticación metodológica como la genética, la biología molecular, la fisiología, la bioquímica y otras como la ficoquímica, la biología celular y la ultraestructura. Los especialistas de estas disciplinas pueden o no ser a su vez ficólogos, ya que muchos de ellos sólo utilizan las algas como material de investigación. Lo fundamental es que las algas son organismos de gran importancia en el descubrimiento científico. Para muchos investigadores, la taxonomía sigue siendo el punto central de la ficología y a menudo se preguntan: ¿Cómo el biólogo molecular puede publicar su trabajo si no conoce el nombre del organismo que está investigando? Esto es cierto sin duda, ya que existen muchas especies que permanecen sin ser aun descritas, especialmente entre las microalgas y las macroalgas de regiones tropicales. Sin embargo, en aquellos grupos relativamente bien conocidos están surgiendo nuevas investigaciones a través de su revaloración, utilizando modernas metodologías (South y Whittick, 1987).

\section{LA FICOLOGÍA EN MÉXICO}

\section{Las disciplinas y su infraestructura}

Al realizar una revisión rápida de los foros científicos nacionales relacionados con la ficología de la última década de este siglo, observamos que los estudios florísticos permanecen aun como punto central de investigación de los ficólogos mexicanos. No obstante, existen otras áreas que se están desarrollando: la morfología - de organismos en crecimiento y adultos -, la ecología y la ficología aplicada en sus diversos aspectos (extracción de ficocoloides, actividad biológica, cultivo de algas e impacto y contaminación ambiental). En el Cuadro 1 se presenta una relación de instituciones, disciplinas, grupos y áreas geográficas de estudio relacionadas con la ficología nacional. 
PHYCOLOGIA

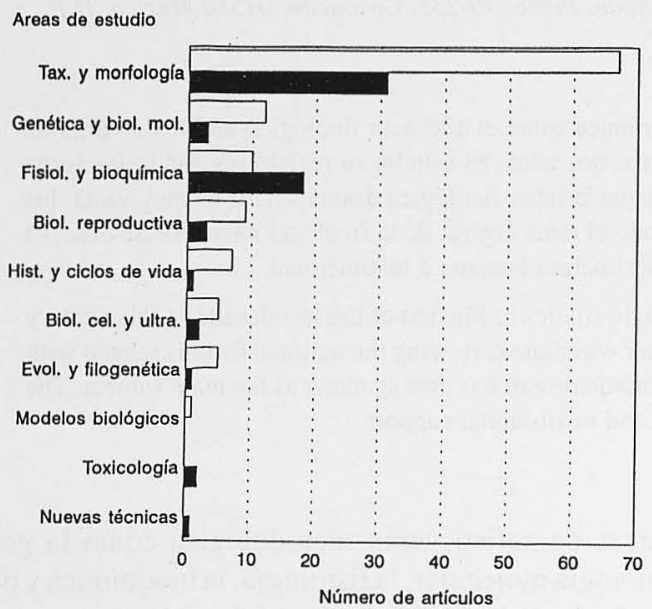

BOTANICA MARINA

\section{Areas de estudio}

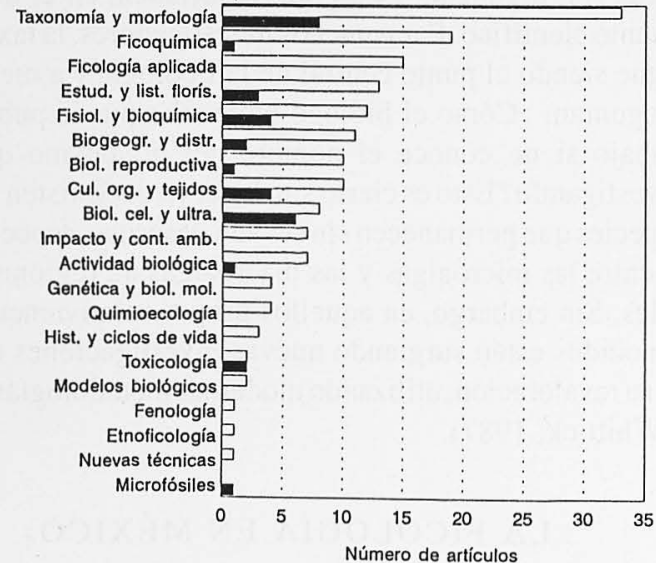

JOURNAL OF PHYCOLOGY
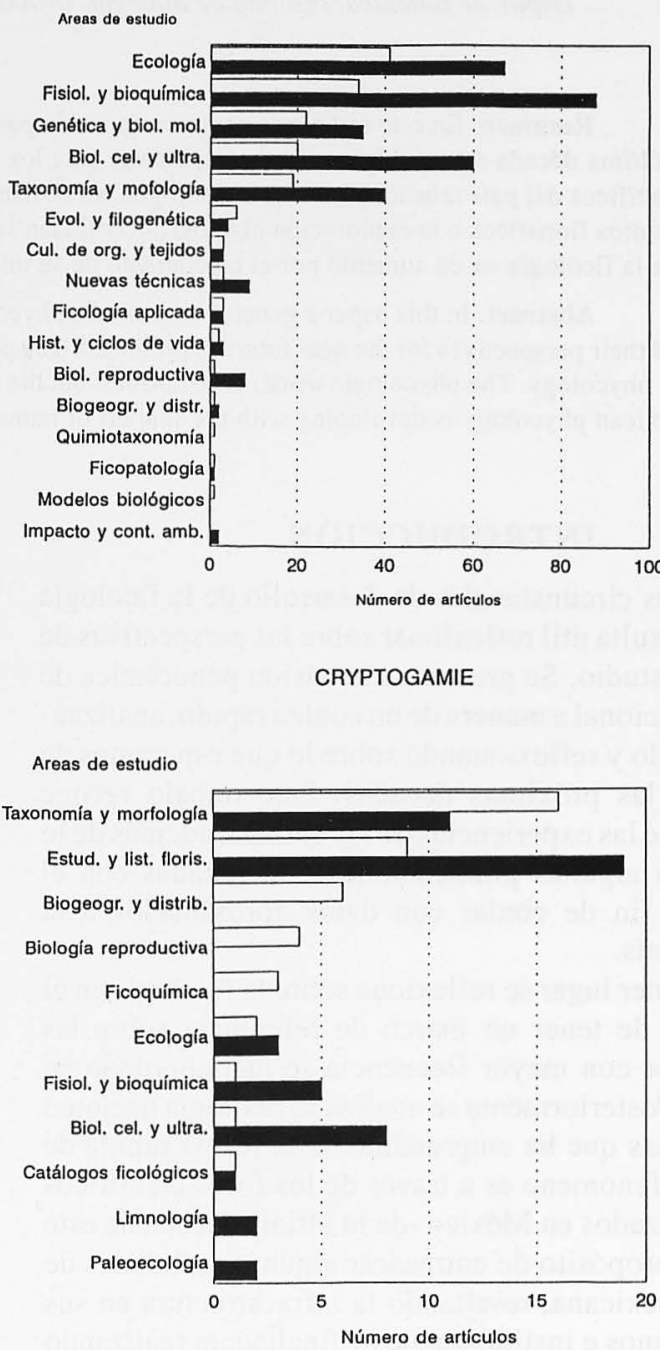

\section{$\square$ Macroalgas}

Microalgas

FIGURA 1. Frecuencia de disciplinas que abordan el estudio de las algas, a través de algunas revistas ficológicas de amplia circulación (1990-1994). 
CuADro 1. Instituciones, disciplinas, grupos y áreas geográficas de estudio de la ficología en México, visto a través de algunos foros ficológicos nacionales recientes (Ortega, 1987; Arreguín Sánchez et al., 1994; XI y XII congresos mexicanos de botánica, 1990, 1993; III Congreso Latinoamericano, 1993).

\begin{tabular}{|c|c|c|c|c|}
\hline EDO. & INSTITUCIÓN & DISCIPLINA & TAXA & ÁREA DE ESTUDIO \\
\hline \multirow[t]{5}{*}{ B.C.N. } & $\begin{array}{l}\text { Centro Interdisciplinario } \\
\text { de Ciencias Marinas, I.P.N. }\end{array}$ & Ecología & Macroalgas (Sargassum) & Península de B.C. \\
\hline & $\begin{array}{l}\text { Centro de Investigación } \\
\text { Científica y de Educación } \\
\text { Superior de Ensenada (CICESE) }\end{array}$ & Ecología & Microalgas & Península de B.C. \\
\hline & $\begin{array}{l}\text { Centro Regional } \\
\text { de Investigaciones } \\
\text { Pesqueras de Ensenada. }\end{array}$ & $\begin{array}{l}\text { Ficología aplicada } \\
\text { (ficocoloides, planta piloto) }\end{array}$ & $\begin{array}{l}\text { Macroalgas } \\
\text { (Macrocystis, Gelidium } \\
\text { y Gigartina) }\end{array}$ & Península de B.C. \\
\hline & $\begin{array}{l}\text { Instituto de Investigaciones } \\
\text { Oceanológicas, } \\
\text { Univ. Autón de B.C. }\end{array}$ & $\begin{array}{l}\text { Estudios y listados } \\
\text { florísticos, citología, } \\
\text { ecología, ficología aplicada }\end{array}$ & $\begin{array}{l}\text { Macroalgas } \\
\text { (Gigartina, Cystoseiraceae, } \\
\text { Phaeophyta, Chlorophyta) }\end{array}$ & Península de B.C., Q.R. \\
\hline & $\begin{array}{l}\text { Facultad de Ciencias, } \\
\text { Univ. Autón. de B.C. }\end{array}$ & $\begin{array}{l}\text { Florística, ficología } \\
\text { aplicada (fertilizantes) }\end{array}$ & Macroalgas & Península de B.C. \\
\hline \multirow[t]{2}{*}{ B.C.S. } & $\begin{array}{l}\text { Centro de Investigaciones } \\
\text { Biológicas de B.C.S. }\end{array}$ & $\begin{array}{l}\text { Ficología aplicada, } \\
\text { cultivo de microalgas }\end{array}$ & $\begin{array}{l}\text { Microalgas } \\
\text { (Porphyridium cruentum, } \\
\text { Tetraselmis suecica) }\end{array}$ & \\
\hline & $\begin{array}{l}\text { Centro Interdisciplinario } \\
\text { de Ciencias Marinas } \\
\text { (CICIMAR), I.P.N. }\end{array}$ & $\begin{array}{l}\text { Florística, morfología, } \\
\text { ecología, ficología aplicada } \\
\text { (planta piloto de alginatos) }\end{array}$ & $\begin{array}{l}\text { Macroalgas } \\
\text { (Macrocystis pyrifera, } \\
\text { Sargassum) }\end{array}$ & Península de B.C. \\
\hline \multirow[t]{2}{*}{ Col. } & $\begin{array}{l}\text { Centro Universitario de } \\
\text { Investigaciones Oceanológicas, } \\
\text { Univ. de Colima. }\end{array}$ & $\begin{array}{l}\text { Florística, ultraestructura, } \\
\text { cultivo de algas }\end{array}$ & Microalgas & Col. \\
\hline & $\begin{array}{l}\text { Facultad de Ciencias Marinas, } \\
\text { Univ. de Colima. }\end{array}$ & Cultivo de algas & Microalgas & Col. \\
\hline \multirow[t]{5}{*}{ D.F. } & $\begin{array}{l}\text { Escuela Nacional } \\
\text { de Ciencias Biológicas, } \\
\text { Deptos. de Botánica, } \\
\text { Zoología y Farmacia }\end{array}$ & $\begin{array}{l}\text { Florística, bioquímica, } \\
\text { toxicología, impacto ambiental } \\
\text { y contaminación }\end{array}$ & $\begin{array}{l}\text { Macroalgas y microalgas } \\
\text { (Dinophyceae, Corallinales, } \\
\text { Champiaceae, Dasyaceae, } \\
\text { Dasycladales, } \\
\text { Helminthocladiaceae, } \\
\text { Scytosiphonaceae, } \\
\text { Sargassum } \\
\text { Ankistrodesmus falcatus) }\end{array}$ & $\begin{array}{l}\text { Gto., Mor. Península } \\
\text { de Yucatán, N y S } \\
\text { del Océano Pacífico }\end{array}$ \\
\hline & $\begin{array}{l}\text { Univ. Autón. Metropolitana, } \\
\text { Iztapalapa. }\end{array}$ & $\begin{array}{l}\text { Florística, ecología, } \\
\text { morfología, cultivo de algas, } \\
\text { actividad biológica }\end{array}$ & $\begin{array}{l}\text { Macroalgas y microalgas } \\
\text { (Ceramiales, Gracilariaceae, } \\
\text { Rhodomelaceae, Sargassaceae, } \\
\text { Padina, Dermonema, } \\
\text { Ceratium, Cladophora, Chlorella) }\end{array}$ & $\begin{array}{l}\text { B.C.S., Gto., Oax., } \\
\text { Ver. Huasteca Potosina, } \\
\text { costas tropicales de México. }\end{array}$ \\
\hline & $\begin{array}{l}\text { Univ. Autón. Metropolitana } \\
\text { Xochimilco. }\end{array}$ & $\begin{array}{l}\text { Cultivo de algas, impacto } \\
\text { y contaminación ambiental }\end{array}$ & $\begin{array}{l}\text { Microalgas } \\
\text { (Ankistrodesmus, } \\
\text { Chlorella, Dunaliella tertiolecta) }\end{array}$ & $\begin{array}{l}\text { Edo. de México } \\
\text { (embalses) }\end{array}$ \\
\hline & Centro de Ecología, UNAM. & Ecología & Microalgas & Edo. de México. \\
\hline & $\begin{array}{l}\text { Facultad de Ciencias, } \\
\text { UNAM. }\end{array}$ & $\begin{array}{l}\text { Florística, estructura } \\
\text { y morfología, ecología } \\
\text { (análisis de ambientes, } \\
\text { y comunidades) } \\
\text { ficología aplicada }\end{array}$ & $\begin{array}{l}\text { Macroalgas y microalgas } \\
\text { (Cyanophyta, Rhodophyta, } \\
\text { Corallinales, Chromophyta, } \\
\text { Phaeophyta, Ralfsciaceae) }\end{array}$ & $\begin{array}{l}\text { Col., Gro., Hgo., Jal., Mor., } \\
\text { Nay., Oax., Pue., Q.R., Ver. } \\
\text { (Huasteca Potosina, } \\
\text { Cuencas de los Ríos Pánuco, } \\
\text { Amacuzac, } \\
\text { Balsas-Lerma, } \\
\text { Papaloapan, Pacífico } \\
\text { Tropical Mexicano) }\end{array}$ \\
\hline
\end{tabular}


CuAdrol. (Cont.)

\begin{tabular}{|c|c|c|c|c|}
\hline EDO. & INSTITUCIÓN & DISCIPLINA & TAXA & ÁREA DE ESTUDIO \\
\hline & $\begin{array}{l}\text { Facultad de Estudios Superiores, } \\
\text { Zaragoza, UNAM. }\end{array}$ & Florística & Macroalgas (Phaeophyta) & Costas de México \\
\hline & $\begin{array}{l}\text { Instituto de Biología, UNAM., } \\
\text { Deptos. Botánica y Zoología }\end{array}$ & $\begin{array}{l}\text { Catálogo de algas marinas } \\
\text { bentónicas, claves de campo, } \\
\text { ficoquímica, estudios sobre algas } \\
\text { continentales, fitoplancton }\end{array}$ & Macroalgas y microalgas & Costa Altántica de México \\
\hline & $\begin{array}{l}\text { Instituto de Ciencias del Mar } \\
\text { y Limnología, UNAM. }\end{array}$ & $\begin{array}{l}\text { Taxonomía y morfología, } \\
\text { florística, ecología } \\
\text { y distribución }\end{array}$ & Microalgas (fósiles y recientes) & $\begin{array}{l}\text { Mar de Cortés, } \\
\text { Golfo de México } \\
\text { y Océano Pacífico. }\end{array}$ \\
\hline Jal. & $\begin{array}{l}\text { Facultad de Ciencias Biológicas, } \\
\text { Univ. de Guadalajara }\end{array}$ & Florística & Microalgas & Jal. (Lago de Chapala) \\
\hline Méx. & $\begin{array}{l}\text { Escuela Nacional de Estudios } \\
\text { Profesionales Iztacala, UNAM. }\end{array}$ & $\begin{array}{l}\text { Florística, limnología } \\
\text { y microfósiles }\end{array}$ & Macroalgas y microalgas & $\begin{array}{l}\text { Edo. de Méx., Pue., } \\
\text { Tab., Tlax., Ver. }\end{array}$ \\
\hline Mich. & $\begin{array}{l}\text { Univ. Michoacana } \\
\text { de San Nicolás de Hidalgo }\end{array}$ & Florística & Microalgas & Mich. (Presa de Zicuirán) \\
\hline Mor. & $\begin{array}{l}\text { Instituto Mexicano } \\
\text { de Tecnología del Agua }\end{array}$ & $\begin{array}{l}\text { Impacto y contaminación } \\
\text { ambiental }\end{array}$ & Microalgas & Mor. \\
\hline N.L. & $\begin{array}{l}\text { Facultad de Ciencias Biológicas, } \\
\text { Univ. Autón. de Nuevo León. }\end{array}$ & Florística & Macroalgas & Tamps. \\
\hline Q.R. & $\begin{array}{l}\text { Centro de Investigaciones } \\
\text { de Quintana Roo (CIQRO). }\end{array}$ & Florística & Macroalgas y microalgas & $\begin{array}{l}\text { Mar de Cortés, } \\
\text { Península de Yucatán }\end{array}$ \\
\hline & $\begin{array}{l}\text { Estación Puerto Morelos, } \\
\text { Instituto de Ciencias del Mar }\end{array}$ & Ecología & Macroalgas & Q.R. \\
\hline
\end{tabular}

Instituto de Ciencias del Mar

y Limnología, UNAM.

Sin. Escuela Ciencias del Mar, Univ. Autón. de Sinaloa.

Estación Mazatlán, Instituto de Ciencias del Mar y Limnología, UNAM.

Son. Centro de Investigaciones Científicas y Tecnológicas, Univ. de Sonora.

Tamps. Instituto de Ecología y Alimentos, Univ. Autón. de Tamaulipas.

Ver. Facultad de Biología, Univ. Veracruzana campus Xalapa.

Yuc. Centro de Investigación y de Estudios Avanzados, Depto. de Recursos del Mar, Unidad, I.P.N. Mérida.

Florística-ecología, ficología aplicada

Florística, toxicología

Cultivo de algas

Florística, ficología aplicada (alimento de aves)

Florística

Florística, ecología
Macroalgas

(Ulva lactuca y Padina durvillaei)

Cyanophyceae, Dinophyceae

Microalgas

Son. (estanques)

Macroalgas y microalgas

Tamps.

Macroalgas

Ver.

Yuc. (Laguna de Celestun)

B.C., Sin.

Sin. (estanques) 
Hay casi 30 instituciones de 14 estados del país que desarrollan algún tipo de investigación ficológica en México, entre las que destacan las del Distrito Federal y Baja California. Esto nos indica que la ficología nacional se encuentra en pleno auge de exploración, pero al mismo tiempo se ocupa de temas de preocupación actual: la explotación de los recursos algales y la contaminación. Muchas de las instituciones cuentan con la infraestructura básica para desarrollar la actividad central de esta área de estudio - la exploración -, como son microscopios estándar y estereoscópicos, cámaras de dibujo y fotomicrografía, equipo de campo, vehículos (terrestres y acuáticos), reactivos, equipo para análisis químico y, recientemente, el uso de las computadoras. Muy pocas instituciones cuentan con microscopios electrónicos (transmisión y barrido).

El mayor número de personas - de diferentes niveles de estudios - relacionadas con la ficología se encuentran distribuidas en mayor proporción en el Distrito Federal, Baja California y Baja California Sur (Fig. 2). Pero en otros estados del país comienza a incrementarse ya la presencia de personal capacitado. Otro aspecto que nos indica el progreso de la ficología es el nivel de estudios de los ficólogos mexicanos. Se observa que la mayoría de personas dedicadas a esta área cuentan con estudios de licenciatura (biolo- gía), pero sólo el $17 \%$ cuenta con maestría y un $6.4 \%$ con doctorado, sin olvidar un buen porcentaje de estudiantes que se encuentran en formación. Lo fundamental es que en esta última década los estudios de grado se han incrementado, y esto refleja que la ficología comienza a tener un lugar importante en la vida científica del país.

No dejaremos de lado la enseñanza, ya que jugará un papel importante en el desarrollo de la ficología en los próximos años. Hasta el momento no se cuenta con una especialidad en ficología en los niveles de maestría o doctorado, pero algunas universidades como la Nacional Autónoma de México y la Autónoma de Baja California incluyen de manera general el estudio de las algas (Dirzo y Cueva del Castillo, 1994). Recientemente, la Universidad Autónoma Metropolitana acreditó un programa de doctorado sobre ciencias, en donde su plan de estudios puede orientarse hacia esta especialidad.

\section{Los herbarios y bibliotecas}

En el trabajo de Ortega (1987) se compilaron los herbarios ficológicos mexicanos que existían hasta 1983. Actualmente observamos un crecimiento sustancial, principalmente en el aumento de sus colecciones, y una tendencia

Figura 2: Frecuencia de personas relacionadas con la ficología de México, vista a través de algunos foros nacionales recientes (XI y XII Congr. Mex. Botánica, 1990, 1993; Ficología...; Arreguín et al., 1994

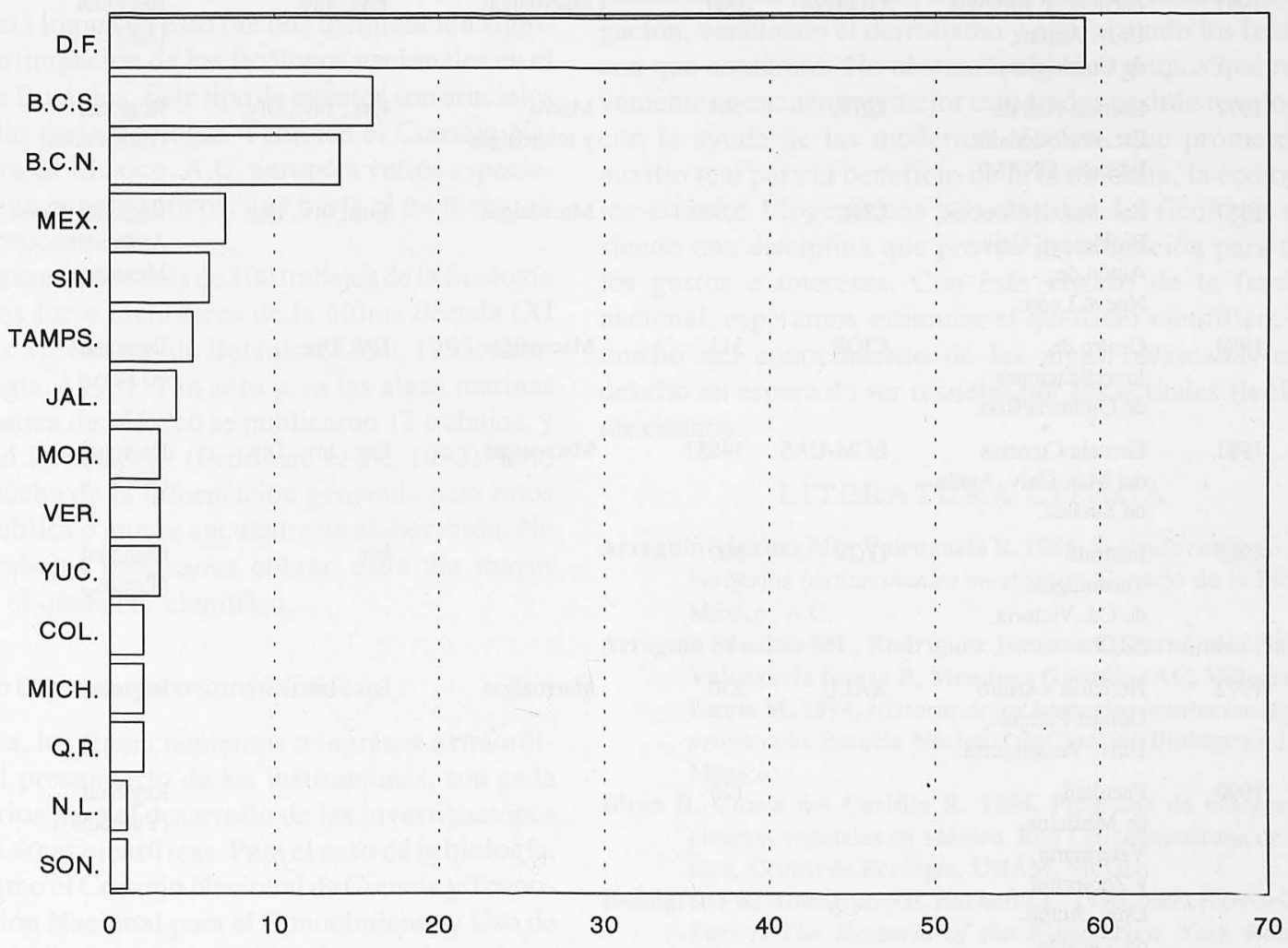


CuAdro 2. Los herbarios ficológicos mexicanos (Ortega, 1987; Arreguín Sánchez y Valenzuela, 1986; Holmgreen et al., 1990; Arreguín Sánchez et al., 1994).

\begin{tabular}{|c|c|c|c|c|c|c|c|c|}
\hline EDO. & FUND. & INSTITUCIÓN & SIGLAS & NO. EJEM. & ESPECIALIC. & USO & ALCANCE & CURADOR \\
\hline \multirow[t]{2}{*}{ B.C.N. } & 1972 & $\begin{array}{l}\text { Facultad } \\
\text { de Ciencias } \\
\text { Marinas, Univ. Autón. } \\
\text { de Baja California. }\end{array}$ & CMMEX & 5,500 & Macroalgas & Ens., Inv., Div. & $\begin{array}{l}\text { Regional } \\
\text { (B.C.) }\end{array}$ & $\begin{array}{l}\text { Ocean. Raúl } \\
\text { Aguilar Rosas. }\end{array}$ \\
\hline & 1981 & $\begin{array}{l}\text { Facultad } \\
\text { de Ciencias } \\
\text { Biol., Univ. Autón. } \\
\text { de Baja California. }\end{array}$ & BCMEX & 1,500 & Macroalgas & Ens., Inv. & $\begin{array}{l}\text { Regional } \\
\text { (B.C.) }\end{array}$ & $\begin{array}{l}\text { MC. Eusebio } \\
\text { Barreto Estrada. }\end{array}$ \\
\hline \multirow[t]{5}{*}{ D.F. } & 1929 & $\begin{array}{l}\text { Herbario Nacional } \\
\text { de México, Instituto } \\
\text { de Biología, UNAM. }\end{array}$ & MEXU & 3,400 & $\begin{array}{l}\text { Macro } \\
\text { y microalgas }\end{array}$ & Inv. & Nacional & $\begin{array}{l}\text { Dra. Martha } \\
\text { M. Ortega. }\end{array}$ \\
\hline & 1938 & $\begin{array}{l}\text { Facultad de Ciencias, } \\
\text { UNAM. }\end{array}$ & FCME & 1,859 & $\begin{array}{l}\text { Macro } \\
\text { y microalgas }\end{array}$ & Ens. Inv., Div. & Nacional & $\begin{array}{l}\text { MC. Hilda } \\
\text { León Tejera. }\end{array}$ \\
\hline & 1943 & $\begin{array}{l}\text { Escuela Nacional } \\
\text { de Ciencias } \\
\text { Biológicas, I.P.N. }\end{array}$ & ENCB & 10,000 & $\begin{array}{l}\text { Macro } \\
\text { y microalgas }\end{array}$ & Ens., Inv., Div. & Nacional & $\begin{array}{l}\text { Biól. Catalina } \\
\text { Mendoza González. }\end{array}$ \\
\hline & $1981-89$ & $\begin{array}{l}\text { Univ. Autón. } \\
\text { Metropolitana, } \\
\text { Unidad Iztapalapa }\end{array}$ & UAMIZ & 700 & Macroalgas & Ens., Inv., Div. & Nacional & $\begin{array}{l}\text { Biól. Kurt } \\
\text { M. Dreckmann. }\end{array}$ \\
\hline & 1982 & $\begin{array}{l}\text { Facultad de } \\
\text { Estudios Superiores } \\
\text { Zaragoza, UNAM. }\end{array}$ & FESZA & 60 & Macroalgas & Ens., Inv., Div. & $\begin{array}{l}\text { Regional } \\
\text { (O. Pacífico) }\end{array}$ & $\begin{array}{l}\text { MC. Alejandrina } \\
\text { G. Avila Ortiz. }\end{array}$ \\
\hline \multirow[t]{2}{*}{ Jal. } & 1960 & $\begin{array}{l}\text { Instituto de Botánica, } \\
\text { Univ. de Guadalajara. }\end{array}$ & IBUG & 135 & Macroalgas & Ens. & $\begin{array}{l}\text { Regional } \\
\text { (Jal.) }\end{array}$ & $\begin{array}{l}\text { Prof. Luz Ma. } \\
\text { Villarreal de Puga. }\end{array}$ \\
\hline & 1967 & $\begin{array}{l}\text { Escuela de Biología, } \\
\text { Univ. Autón. } \\
\text { de Guadalajara. }\end{array}$ & GUADA & 600 & Macroalgas & Ens., Inv. & $\begin{array}{l}\text { Regional } \\
\text { (Jal.) }\end{array}$ & $\begin{array}{l}\text { Biól. Leopoldo } \\
\text { Villarruel S. }\end{array}$ \\
\hline Méx. & 1977 & $\begin{array}{l}\text { Escuela Nal. de } \\
\text { Est. Profesionales, } \\
\text { Iztacala, UNAM. }\end{array}$ & IZTA & 504 & $\begin{array}{l}\text { Macro } \\
\text { y microalgas }\end{array}$ & Ens., Inv., Div. & $\begin{array}{l}\text { Regional } \\
\text { (varios edos) }\end{array}$ & $\begin{array}{l}\text { Biól. Gloria } \\
\text { Garduño Solórzano. }\end{array}$ \\
\hline N.L. & 1957 & $\begin{array}{l}\text { Facultad de Ciencias } \\
\text { Biológicas, Univ. } \\
\text { Autón.de } \\
\text { Nuevo León. }\end{array}$ & UNL & 5,000 & Macroalgas & Ens., Inv., Div. & $\begin{array}{l}\text { Regional (Costa } \\
\text { Atlántica } \\
\text { Mexicana) }\end{array}$ & $\begin{array}{l}\text { MC. Salomón } \\
\text { Martínez Lozano. }\end{array}$ \\
\hline Q.R. & 1984 & $\begin{array}{l}\text { Centro de } \\
\text { Investigaciones } \\
\text { de Quintana Roo. }\end{array}$ & CIQR & 311 & Macroalgas & Inv., Div. & $\begin{array}{l}\text { Regional } \\
\text { (Q.R.) }\end{array}$ & $\begin{array}{l}\text { MC. Julio } \\
\text { Espinoza. }\end{array}$ \\
\hline Sin. & 1981 & $\begin{array}{l}\text { Escuela Ciencias } \\
\text { del Mar, Univ. Autón. } \\
\text { de Sinaloa. }\end{array}$ & ECM-UAS & $1485 ?$ & Macroalgas & Ens., Inv., Div. & $\begin{array}{l}\text { Regional } \\
\text { (Sin.) }\end{array}$ & $\begin{array}{l}\text { Biól. Julia } \\
\text { Ochoa I. }\end{array}$ \\
\hline Tamps. & 1985 & $\begin{array}{l}\text { Instituto } \\
\text { Tecnológico } \\
\text { de Cd. Victoria, } \\
\text { S.E.P. }\end{array}$ & ITCV & 300 & & Inv. & $\begin{array}{l}\text { Regional } \\
\text { (Tamps.) }\end{array}$ & $\begin{array}{l}\text { Biól. Jesús } \\
\text { García Jiménez } \\
\text { (encargado). }\end{array}$ \\
\hline Ver. & 1972 & $\begin{array}{l}\text { Herbario «Arturo } \\
\text { Gómez Pompa», } \\
\text { Univ. Veracruzana. }\end{array}$ & XALU & 250 & Macroalgas & Ens., Inv. & Regional (Ver.) & $\begin{array}{l}\text { Biól. Roberto } \\
\text { Blanco. }\end{array}$ \\
\hline Yuc. & 1990 & $\begin{array}{l}\text { Facultad } \\
\text { de Medicina, } \\
\text { Veterinaria } \\
\text { y Zootecnia, } \\
\text { Univ. Autón. } \\
\text { de Yucatán. }\end{array}$ & & 125 & & & $\begin{array}{l}\text { Regional } \\
\text { (Yucatán) }\end{array}$ & $\begin{array}{l}\text { Q.F.B. Ma. Isabel } \\
\text { Sánchez M. }\end{array}$ \\
\hline
\end{tabular}


hacia la regionalización aunque existen otros proyectos de alcance nacional (Cuadro 2). Es necesario aclarar que algunos herbarios cuentan con colecciones de otras regiones del mundo y con colecciones particulares. Por ejemplo, el MEXU cuenta con la Phycoteca Boreali Americana; no obstante, su acervo principal se enfoca hacia los especímenes mexicanos. El mayor número de ejemplares herborizados consultables se encuentra en instituciones del Distrito Federal y de los estados de Baja California y Nuevo León.

No es menor la importancia de las bibliotecas para el desarrollo de la ficología. Por muchos años, las principales bibliotecas, incluso las personales, estuvieron incorporadas a la Universidad Nacional Autónoma de México y la Escuela Nacional de Ciencias Biológicas del I.P.N. Actualmente, con el incremento de los servicios electrónicos hacia los bancos de información mundial, incluyendo bibliotecas complementarias como las mapotecas, este problema ha disminuido. Esto no quiere decir que las actuales bibliotecas sean autosuficientes, pero sí resulta más fácil hoy en día conseguir algún tipo de bibliografía.

\section{La difusión de la información ficológica}

En la década pasada, la ficología nacional contaba con un único foro nacional, el Congreso Mexicano de Botánica organizado por la Sociedad Botánica de México, S.C., en donde la ficología ocupaba un lugar importante. No obstante, en esta década se organizó el Ier Congreso Mexicano de Ficología coordinado por la Sociedad Ficológica de México. Una consecuencia lógica de esto fue una disminución significativa en la participación de los ficólogos nacionales en el XII Congreso de Botánica. Este tipo de eventos son cruciales para el desarrollo de la ficología. También el Consejo Nacional de la Flora de México, A.C. agrupó a varios especialistas para trabajos monográficos, que hasta el momento se encuentran en proceso.

Fueron presentados más de 100 trabajos de la ficología nacional en estos foros científicos de la última década (XI y XII Congresos Mexicanos de Botánica, 1990, 1993; Congreso de Ficología, 1993). Tan sólo para las algas marinas de la costa Atlántica de México se publicaron 12 trabajos, y para la costa del Pacífico 19 (Pedroche et al., 1993). Esto significa que mucha de la información generada para estos eventos no se publica o aun se encuentra en elaboración. No obstante, los trabajos mexicanos cobran cada día mayor importancia en el quehacer científico.

\section{Los subsidios o ingresos extraordinarios}

Hoy en día, los financiamientos o ingresos extraordinarios ajenos al presupuesto de las instituciones, son cada día más necesarios para el desarrollo de las investigaciones en casi todas las áreas científicas. Para el caso de la biología, instituciones como el Consejo Nacional de Ciencia y Tecnología, la Comisión Nacional para el Conocimiento y Uso de la Biodiversidad -de reciente creación-, las propias univer- sidades, así como empresas del sector privado, proveen de ayudas financieras a proyectos originales de investigación mediante previas evaluaciones y engorrosos trámites administrativos. Esta es una realidad que seguirá presente en los siguientes años.

\section{CONSIDERACIONES FINALES}

Realizar un análisis de las perspectivas de la ficología nacional no es una tarea fácil, pero podemos anotar algunas reflexiones.

Las disciplinas prioritarias o que requerirán mayor atención en el futuro son la taxonomía y el reforzamiento de los estudios florísticos, pues existen todavía áreas sin explorar y muchas especies aun no se han descrito. El estudio de pequeñas floras regionales o de algunos grupos particulares puede ser una estrategia que cobrará importancia en los años futuros. Al respecto Silva (1984) sugiere, para el caso de los taxónomos, no olvidar todo el contexto en que nos encontramos trabajando, es decir, considerar factores como el tiempo y lugar, la secuencia histórica -la competencia relacionada con la habilidad, experiencia, interés y objetivo personal-, la estética -ya que un taxónomo es propiamente un artista-, la tradición, la didáctica -referente a la superación en la exposición de ideas- y el uso cotidiano de la ejemplificación.

Una pregunta que por el momento no tiene respuesta es: ¿qué está ocurriendo con la flora ficológica de México? Existen muchos retos para el ficólogo en el futuro, pero lo fundamental es fraccionar un gran problema en pequeños problemas que resuelvan, a corto plazo, un tema de investigación, venciendo el derrotismo y optimizando los recursos con que contamos. No obstante, algunos grupos que relativamente se encuentran mejor estudiados podrán revalorarse con la ayuda de las modernas técnicas que prometen un auxilio real para el beneficio de la taxonomía, la ecología y los estudios filogenéticos y evolutivos. La ficología sigue siendo una disciplina que provee investigación para todos los gustos e intereses. Con este vistazo de la ficología nacional, esperamos estimular el quehacer científico, pues mucho del conocimiento de las algas mexicanas es un desafío en espera de ser resuelto por los actuales ficólogos mexicanos.

\section{LITERATURA CITADA}

Arreguín Sánchez ML, Valenzuela R. 1986. Segundo catálogo de los herbarios institucionales mexicanos. Consejo de la Flora de México, A.C.

Arreguín Sánchez ML, Rodríguez Jiménez C, Fernández Nava R, Valenzuela Garza R, Mendoza González AC, Villegas y de Gante M. 1994. Historia de los herbarios institucionales y su proyección. Escuela Nacional de Ciencias Biológicas, I.P.N., México.

Dirzo R, Cueva del Castillo R. 1994. Programa de postgrado en ciencias vegetales en México. Red Latinoamericana de Botánica, Centro de Ecología, UNAM, México.

Holmgren PK, Holmgren NH, Barnett LC. 1990. Index Herbariorum. Part I: The Herbaria of the World. New York Botanical Garden, Bronx. 
Ortega MM. 1987. Doce años de ficología en México (1971-1983). En: Gómez Aguirre S, Arenas Fuentes V, edrs. Contribuciones en hidrobiología. UNAM, México, 55-186.

Pedroche FF, Dreckmann KM, Sentíes A, Margaín Hernández R. 1993. Diversidad algal en México. Rev. Soc. Mex. Hist. Nat. 44 (vol. esp.): 69-92.
Silva PC. 1984. The role of extrinsic factors in the past and future of green algae systematics. En: Irvine DEG, John DM, edrs. Systematic of the green algae. Academic Press, Londres, 419 433.

South RG, Whittick A. 1987. Introduction of phycology. Blackwell Scientific Publications, Oxford. 